Vol. 6, Num. 1, 2021

\title{
RECOMMENDATION DIALOG SYSTEM FOR SELECTING THE COMPUTER HARDWARE CONFIGURATION
}

\author{
Oksana Svystun, Iryna Yurchak \\ Lviv Polytechnic National University, 12, Bandera Str, Lviv, 79013, Ukraine. \\ Authors'e-mail: oksana.svystun.ki.2017@lpnu.ua, \\ iryna.y.yurcak@lpnu.ua
}

Submitted on 04.05.2021

(C) Svystun O., Yurchak I., 2021

\begin{abstract}
The development of modern technologies is extremely fast. Every day more and more new and various means appear in the world to improve the quality of life and more. It is not possible for a person to process all this new information on the scale and speed with which this information appears. Everyone has their own preferences and wants to receive and obtain information about certain events or things that they are interested in. This has become one of the most important reasons for creating referral systems.

The purpose of developing a recommended dialog system for selecting the computer hardware configuration is to help users choose the computer's hardware characteristics to suit their requirements and needs. This system is suitable for being used by both qualified users in this field, and for users unfamiliar with computer technology.

There has been an attempt to analyze the types of recommendation dialog systems and their varieties in the paper. The principle of operation of the recommendation dialog system in the form of a chat bot made on the platform of the messenger Telegram has been considered.
\end{abstract}

Index Terms: recommendation system, dialog system, chat bot, Telegram, computer hardware configuration.

\section{INTRODUCTION}

Modern search engines provide a user with much more information than he is able to comprehend and process. Because of this, a user may simply get lost in the search and not get what he wanted. This is where recommendation systems come to the rescue. The user explicitly or implicitly provides such systems with information about their preferences and desires, and referral systems filter the amount of information provided by search engines

Recommendation systems are designed to provide guidance to a user on the choice of subjects that interest them. Subjects can be a variety of things - services, goods, objects, or any other objects of interest to a user.

One of the subtypes of referral systems in which a user explicitly sets the criteria for the desired result are referral dialog systems or chatbots. People are often inclined to such systems, because they implement the concept of two-way communication, i.e., dialogue, which provides advantages over other systems. Typically, chatbots use machine learning to identify patterns of communication. Through constant interaction with people, they learn real conversation and respond to oral or written requests, helping to find answers. Modern advanced chatbots use artificial intelligence, therefore, understand the meaning of the conversation, not just commands. Thus, after each dialogue, they become smarter.

A significant proportion of chatbots operate on the basis of programmed scenarios with multiple choices, for example, option A leads to option B and so on. Such chatbots are usually used in referral systems. Recommendation systems are designed to provide guidance to the user on the choice of subjects that interest them. Subjects can be a variety of things - services, goods, objects, or any other objects of interest to the user.

\section{THE RATIONALE OF THE NEED FOR RECOMMENDATION DIALOG SYSTEM FOR SELECTING THE COMPUTER HARDWARE CONFIGURATION}

Researching have shown that telephones are used for messaging more often than for other purposes [1]. Research authors [2] note that messengers are currently used by users around the world for solving a variety of tasks that go beyond simple text messaging framework, as well as for customer interaction with companies, finding the right products, content consumption and others.

The recommendation dialog system will allow a user to configure the computer according to needs and requirements. The system will determine the general characteristics of the computer that fits the requirements of a user and introduce some modern implementations such requirements. Since such a system was not detected, this chat bot will be useful, because receiving recommendations in the form of dialogue is becoming increasingly popular and familiar to Internet users.

The relevance of the final qualifying work is due to the high popularity of messengers and automation tools such as chatbots among Internet users. Chatbots allow to implement daily routine tasks such as weather information, traffic jams, breaking news and more. The main advantage over conventional applications is that one can combine all the features on a single messenger platform. 


\section{SYSTEM COMPONENTS}

\section{A. RECOMMENDATION SYSTEM}

Recommendation system - a subclass of the system that removes redundant information from the information flow using automatic (semi-automatic) or computer methods and provides it to the human user. It defines a list of objects that the user can prefer. The main task of such systems is to filter out redundant information so that the user can focus on his interests and needs.

There are two main concepts of creating recommendation systems: collaborative filtering and content filtering [3].

The basic idea behind collaborative filtering (Fig. 1) techniques is that past user interactions with content are sufficient to identify similar users and similar content and predict preference based on that user.

\begin{tabular}{|c|c|c|c|c|}
\multicolumn{1}{c|}{ Blog } & Kate & Tom & \multicolumn{1}{c}{ Sam } & \multicolumn{1}{c}{ Nancy } \\
\hline Linux & 13 & 3 & 11 \\
\hline OpenSourse & 10 & - & - \\
\hline Cloud Computing & 6 & 1 & 9 \\
\hline Java Technology & - & 6 & - \\
\hline Agile & - & 7 & 1 \\
\hline \multicolumn{3}{|c|}{ Articles read per user } \\
\hline Cluster & 1 & 2 & - \\
\hline
\end{tabular}

Fig. 1. Collaborative filtering

Collaborative filtering and its modifications is one of the most commonly used recommendation algorithms. Even data scientist beginners can use it to build their personal movie recommender system, for example, for a resume project.

When we want to recommend something to a user, the most logical thing to do is to find people with similar interests, analyze their behavior, and recommend the same items to our user. Or we can look at the items similar to ones which the user bought earlier, and recommend products which are similar to them [4].

Collaborative filtering is suitable for systems that are based on a model of previous user behavior. The model can be built solely on the behavior of one user or (more effectively) on the behavior of other users who have similar traits. Taking into account the behavior of other users, collaborative filtering uses knowledge of groups to form recommendations based on common features. In essence, the recommendations are based on the automatic cooperation of several users and filtering on those who show similar traits or behavior.

Another method of recommendation systems is content-based filtering (Fig. 2). In this method, a user profile is created to provide information about the types of items that the user likes, based on the keywords used to describe the items. The content-based filtering method creates a profile for each element, which is used to characterize the element in the system. The system then creates a profile for the user based on items that the user has previously rated or purchased and items that the user is viewing [5].

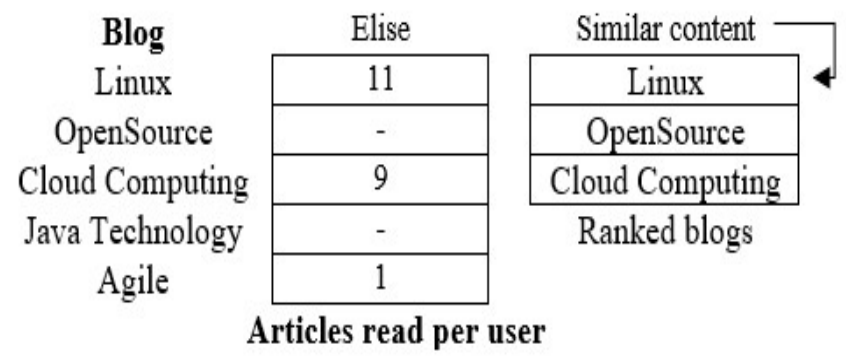

Fig. 2. Content-based filtering

This approach can use browsing history, such as which blogs a user reads and the characteristics of those blogs. If a user frequently reads articles about Linux or is likely to leave comments on software development blogs, contentbased filtering can use this history to identify and recommend similar content.

In both cases this recommendation engine has two steps:

- Find out how many users/items in the database are similar to the given user/item.

- Assess other users/items to predict what grade you would give the user of this product, given the total weight of the users/items that are more similar to this one.

\section{B. DIALOG SYSTEM (CHATBOTS)}

Dialog system - a computer system that uses dialog mode. In this paper, the meaning of the dialog system is the concept of a chatbot, which is a dialog system.

Chatbots are conversational programs that are used to simulate communication with a living person. They appeared many years ago, but have become a trend only now. The revolution took place when the pattern of "communication" changed to the pattern of "recommendations", and, in fact, chatbots became similar to salespeople or consultants [6]. Thus, to date, there are almost perfect chatbots that are able to support a conversation on almost any topic, but still cannot replace a person.

Bots are used to support new users, such as existing ones, who can receive and place orders, collect customers, organize active mailings and much more.

Messengers are the main channel for using bots for both entertainment and business prices. The active audience of messengers is 2 billion people.

You can also use bots to chat on social networks and be able to respond to invitations to events, expand mass comments in groups, automatically receive messages, add subscribers, get likes, and more.

The main advantages of chatbots:

- No waiting time. Customers do not need to wait to talk to a service representative. 
- Minimized real person's mistakes. Rule-driven chatbots classify problems and automatically point them where they need to go.

- Faster solution of various problems. Even when the conversation is transmitted to the person, the chatbot captures all the necessary information in advance so that the person can solve the problem or answer questions faster.

- Improved self-service. Chatbots direct users to the right resources so they can help themselves.

- Cost optimization. Chatbots can reduce staff and automate a variety of customer service processes.

In modern messengers, chatbots are a universal tool for solving various tasks. Bot is a special program that executes any actions through interfaces, designed for people automatically or by a given schedule [7]. Usually, bots are assigned to perform monotonous and repetitive work, at the maximum possible speed. They are used when a faster response is needed more than that of humans. In Fig. 3 one can see a classification of chatbots.

Classification of chatbots

\begin{tabular}{|l|l|}
\hline Classification feature & Type of chatbot \\
\hline User & $\begin{array}{l}\text { Personal } \\
\text { Business }\end{array}$ \\
\hline Interface & $\begin{array}{l}\text { Button } \\
\text { Text }\end{array}$ \\
\hline Access & $\begin{array}{l}\text { Added to group } \\
\text { By subscription } \\
\text { Built-in dialogue }\end{array}$ \\
\hline Purpose & $\begin{array}{l}\text { Communication } \\
\text { Functional }\end{array}$ \\
\hline Working principle & $\begin{array}{l}\text { Template } \\
\text { Learner }\end{array}$ \\
\hline
\end{tabular}

Fig. 3. Cassification of chatbots.

More about Button interface:

The bot works with buttons and commands. It also has a messenger interface, but instead of being able to communicate with the text, the person is offered a choice of categories (or questions / suggestions) that may interest him.

"Communication" occurs by pressing buttons, and the bot responds to them as commands. This chatbot is most reminiscent of a regular mobile application, except that it does not have its own interface, and uses a messenger to work. Commands for the bot are assigned during its creation, and only some platforms allow to change them manually through the admin panel.

Chatbots are especially needed by large businesses, where a lot of resources are spent interacting with customers. These smart assistants take on routine tasks, automate workflows, reduce the workload of the company's employees and significantly reduce the company's costs.

\section{FORMULATION OF THE PROBLEMS}

$51 \%$ of users expect an immediate response from brands at any time of the day. To do this, businesses launch a 24/7 support service and spend a lot of money on it. But most of the user questions are typical, and a properly configured program, for example, a chatbot, may well answer them. And users are satisfied with this: on average, $30-40 \%$ of users respond to chatbots, and well-configured chatbots receive answers in $90 \%$ of cases.

The chatbot creates the feeling that the business is always in touch with you. And if the robot solves all the user's problems, the presence of a person is not needed.

If bots are so profitable, why haven't most businesses implemented them (Fig. 4)? Brands lack examples of the use of chatbots, they worry about the privacy of users and the fact that the bot will give inaccurate answers.

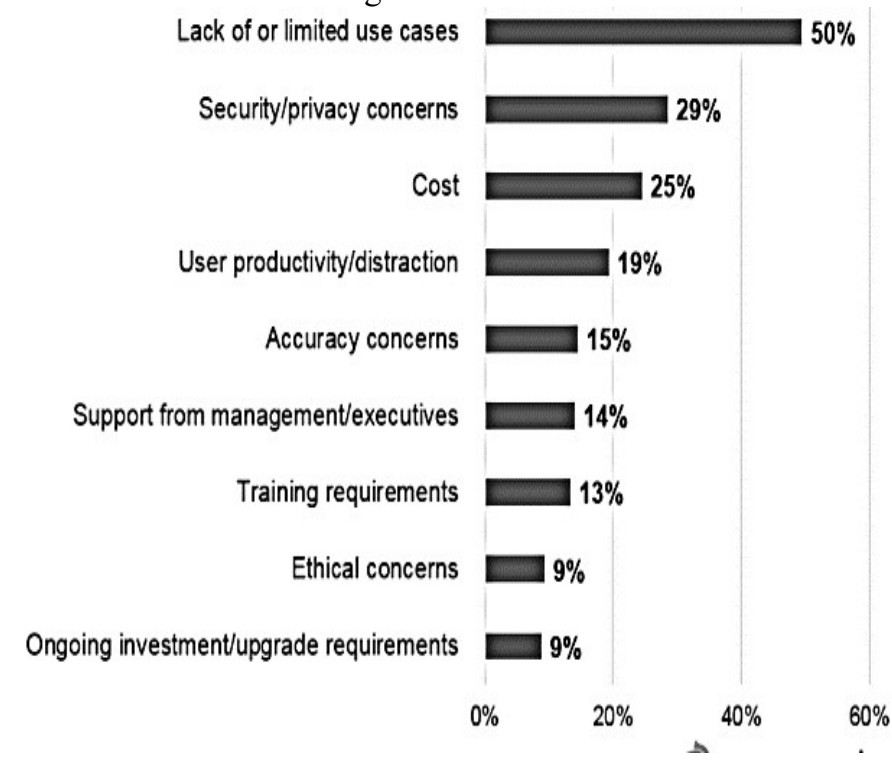

Fig. 4. Top reasons organizations haven't implemented intelligent assistants/chatbots

Therefore, the recommendation dialog system for selecting the computer hardware configuration can become one of these necessary examples of using chatbots.

\section{FUNCTIONS AND FUNCTIONAL PRINCIPLES OF THE RECOMMENDATION DIALOG SYSTEM FOR SELECTING THE COMPUTER HARDWARE CONFIGURATION}

The recommendation dialog system for selecting the computer hardware configuration is not equipped with a large number of different functionalities, because it is narrowly focused, and therefore focuses on the recommendations of the computer's hardware configurations on the data received from the user. Personal computer (PC) configuration - a set of PC components designed to solve certain types of tasks (processing office documents, working with graphics, etc.).

By purpose and, accordingly, by configuration, there are: 
- servers;

- workstations of wide and narrow profile;

- PC for enthusiasts;

- gaming computers;

- multimedia computers;

- general purpose PCs (office, educational);

- $\quad$ laptops (laptops and netbooks, tablets);

- highly specialized systems (in ATMs, in automatic systems, toys, etc.).

- In Fig. 5 one can see a simple example bot script to better understand how they work.

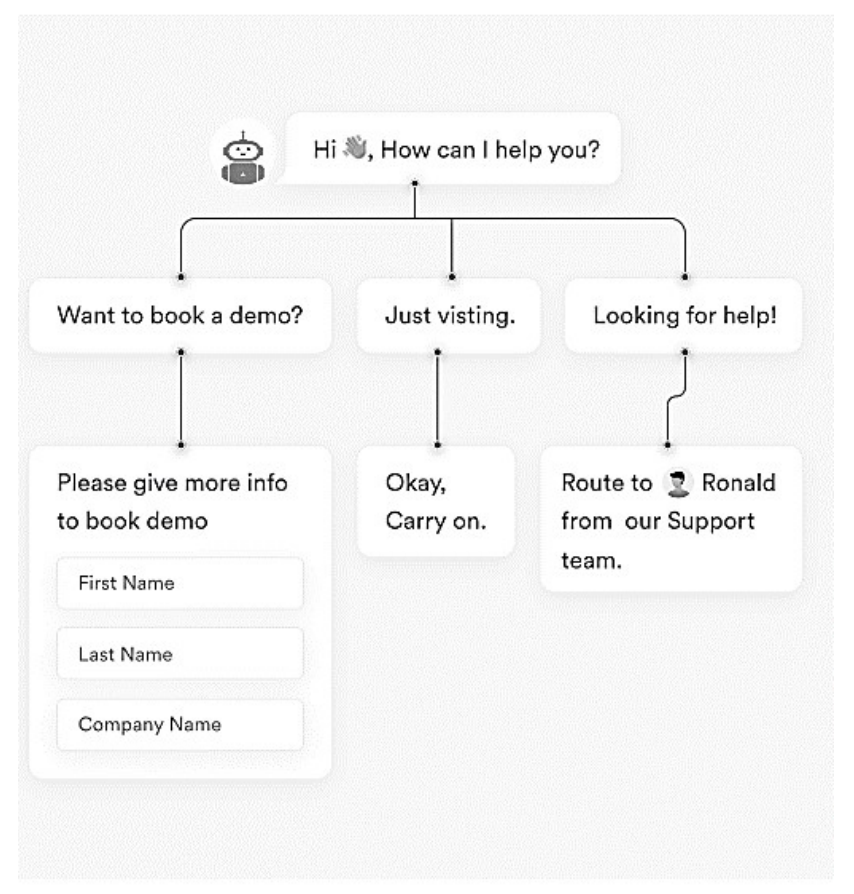

Fig. 5. Example script

Depending on the user's level of knowledge, the system will work in two scenarios: a survey of components and a survey of needs

The system will determine the required configuration according to the following user-specified parameters:

- The total cost

Needed to limit all possible options to a possible price category

- $\quad$ CPUs

The CPU is the core of the computer and ensures the execution of the program stored in RAM. The execution process consists of sequentially reading the program command codes from memory and then executing them.

- RAM

Random access memory - high-speed computer memory designed to write, store and read information during processing.

- Motherboards

- A motherboard is a board that contains the basic components of a computer that provide logic.
- $\quad$ Video cards

A video card is an electronic device, a part of a computer designed to generate and process images and then display them on a peripheral screen.

- Drives

Drive - a computer storage device based on memory chips and controller, which does not contain moving mechanical parts

\section{COMPARATIVE ANALYSIS OF EXISTING RECOMMENDATION SYSTEMS AND CHATBOTS}

Today, many different resources and systems use referral systems. They are used for both general and commercial recommendations. Resources using a variety of referral systems:

- Amazon

Amazon uses content-based recommendations with its own patented Item-To-Item Collaborative Filtering approach

- LinkedIn

Collaborative filtering system based on Apache Hadoop technology is used to form recommendations

\section{- Netflix}

This video service uses a recommendation system based on artificial intelligence and machine learning to show its subscribers personalized trailers of movies and series based on viewers' preferences.

- $\quad$ Last.fm

The service implements the concept of User Generated Content. Users share with the service information about the songs they listen to. In addition, Last.fm uses data about the songs themselves - author, style, date, etc.

To Collaborative filtering we need a different user to recommend content and for Content-based filtering we don't.

Here, one can see the difference between Collaborative filtering (Fig. 6) and Content-based filtering (Fig. 7).

\section{COLLABORATIVE FILTERING}

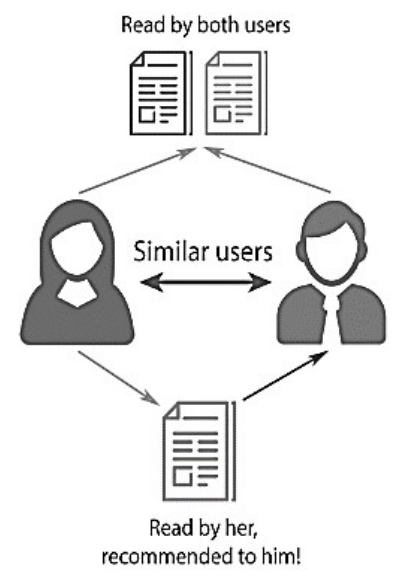

Fig. 6. Difference between Collaborative and Contentbased filtering (a) 
Recommended chat bots can be very useful. They create a sense of communication with a real person. They provide a sense of customer interest. Of course, not in all cases it is advisable to use chat bots for recommendations. Sometimes the best option would be to use an application where the user will choose, or contact a real consultant.

Consider such chat bots that provide recommendations compared to other systems.

\section{CONTENT-BASED FILTERING}

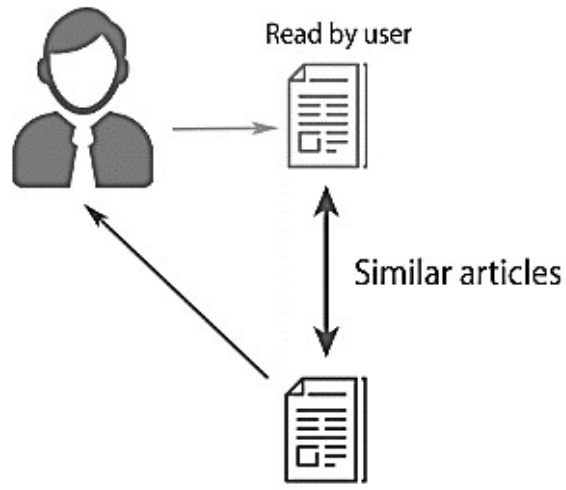

Recommended

to user

Fig. 7. Difference between Collaborative and Contentbased filtering (b)

In Fig. 8 a chatbot that helps pick up a scent and redirects to a purchase on Amazon. Fig. 9 is an application for buying perfume with search filters. Fig. 10 - searching for perfume in the Amazon application. The bot gives a sense of personal touch.

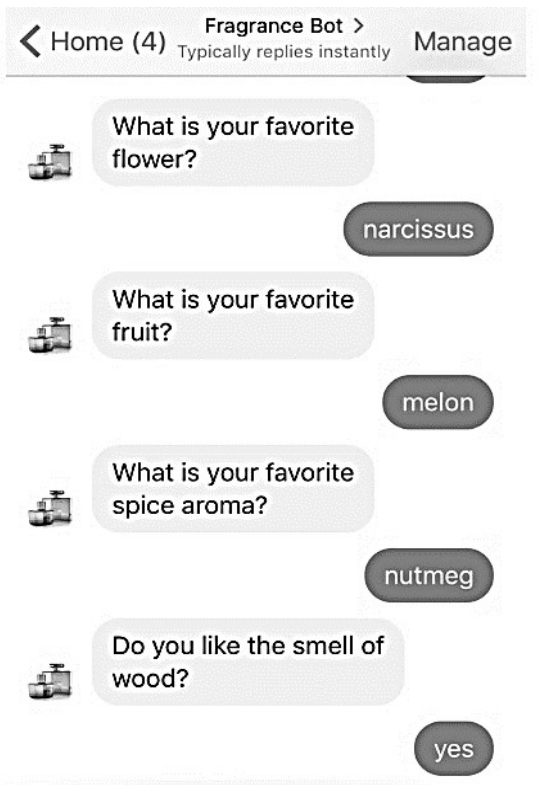

Fig. 8. Chatbot

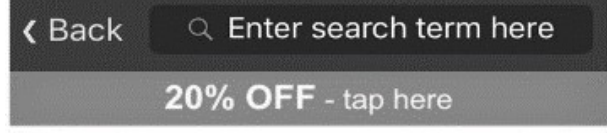

\section{Top Selling Perfumes}

540 ITEMS FOUND

\begin{tabular}{|lr}
\hline \multicolumn{1}{|c|}{ Filter $\bar{\gamma}$} & Sort $\boldsymbol{Y}$ \\
\hline Gender & + \\
\hline Category & + \\
\hline Brand & + \\
\hline Product Type & + \\
\hline Scents/Notes & + \\
\hline
\end{tabular}

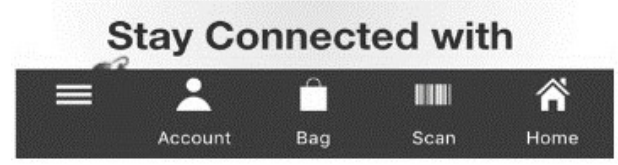

Fig. 9. App with search filters

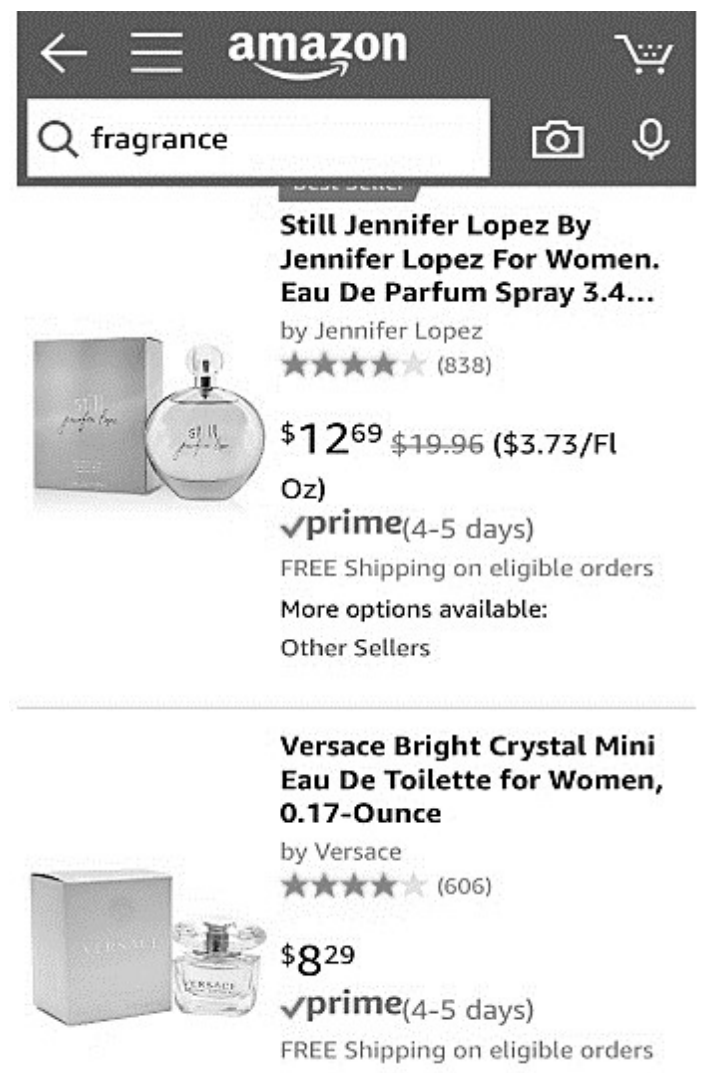

Fig. 10. Amazon app

In this case, the bot gives the feeling of a personal approach. Thus, more consumers will be happy with a 
personal and comfortable approach to choosing a product, rather than a simple search through filters.

On the other hand, the app makes it easier to stand out from the competition due to its eye-catching design.

In addition, with the help of GUI applications there are some things at the moment, everything can be done faster [8].

In the following case, it is better to use the application, because it is even more convenient and fast:

Ordering cinema tickets: in Fig. 11 - a bot, one needs to look in the picture for the row number and seat number, dial them. To order a second ticket, repeat the procedure from the beginning. In Fig. 12 there is an application. One can immediately click on the appropriate places.

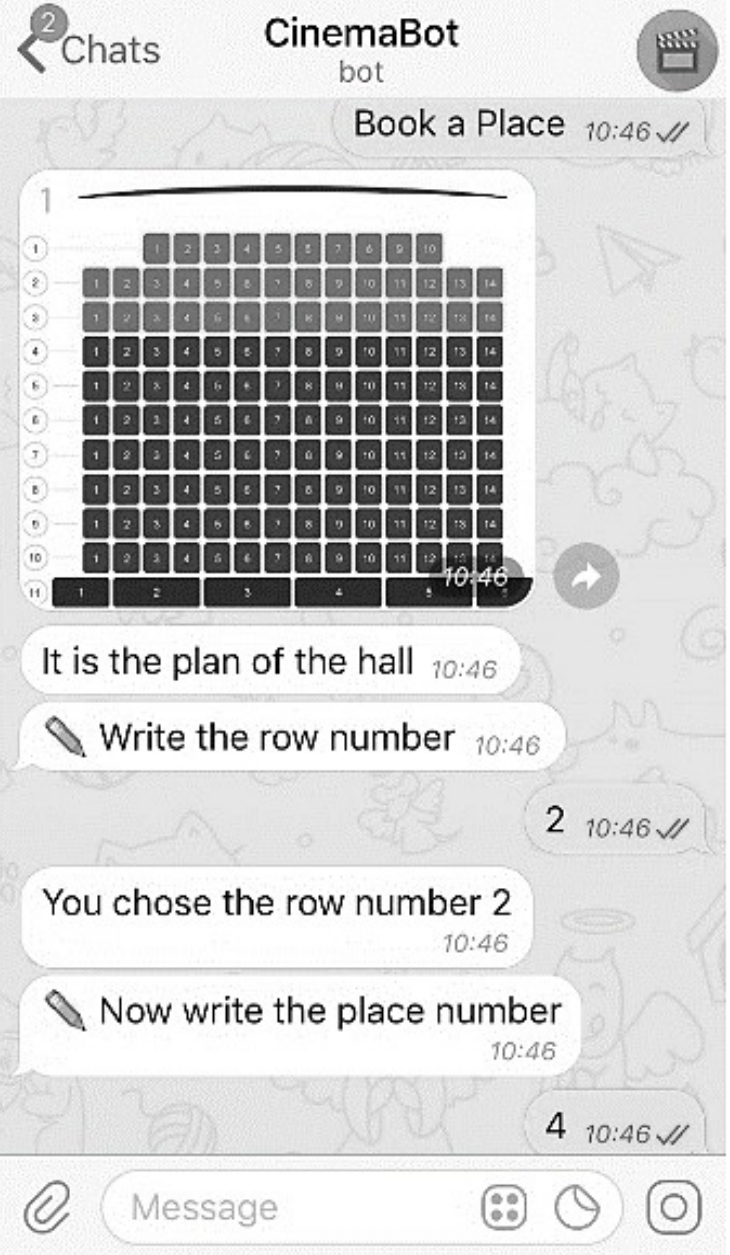

Fig. 11. Ordering cinema tickets in bot

It is very important to understand for what purposes the chat bot will be used and how convenient it will be to use. Well, sometimes a supplement is more appropriate.

The recommendation dialog system for selecting the computer hardware configuration looks like a chat bot, because it is comfortable for a user to communicate or choose certain options to configure the computer. Still simple and clear. It is this simplicity and convenience that the PC will bribe its users.
The recommendation dialog system for selecting the computer hardware configuration will be a good application or any store or portal that sells computers, or their spare parts.

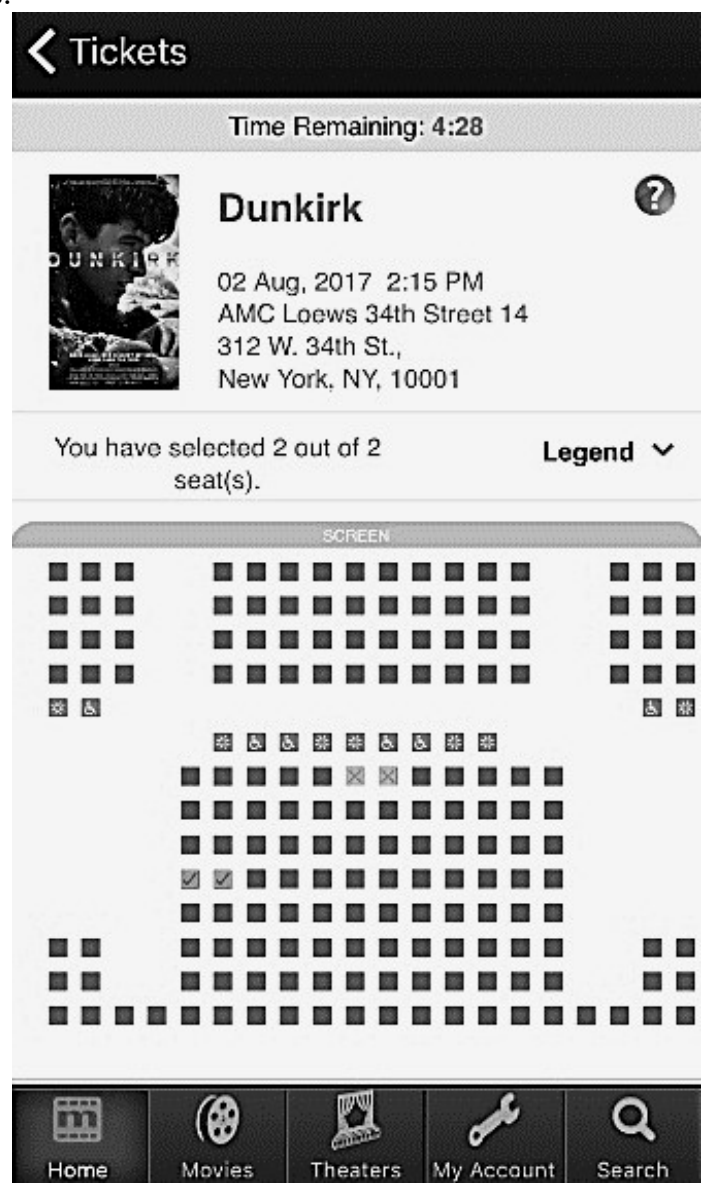

Fig. 12. Ordering cinema tickets in application

\section{TELEGRAM}

Telegram is a free cross-platform cloud messenger for smartphones, tablets and PCs that allows you to exchange text messages and media files, supports voice communication and video conferencing. Founded in 2013 by Russian brothers Nikolai and Pavel Durov.

Telegram has a chatbot platform. Bots can perform a variety of tasks, such as searching the Internet or government registries, shopping, payments, entertainment, group moderation, etc. [9][9]. The Telegram user and a third-party computer program take part in the communication.

Bots are simply Telegram accounts operated by software - not people - and they'll often have AI features [10]. They can do anything - teach, play, search, broadcast, remind, connect, integrate with other services, or even pass commands to the Internet of Things.

The user can interact with the bot using the elements of the messenger interface: sending messages, pressing commands and buttons, using the inline mode. Telegram 
provides three ways for the user to interact with the bot: private chat (classic way), group, and so-called inline mode:

The most common way is private chat. For the most part, the bot cannot initiate a dialogue with the user.

Some bots can be members of groups. For example, in groups, a bot can maintain a conversation, moderate messages, or host a game.

The inline mode resembles a search engine interface. The user enters a query in the message entry field, starting with the short name of the bot. Then the user can select and send one of the results.

Some bots may be members of channels. In the channels bots are used mostly for the planned placement of messages. The user interacts with such bots via a private chat or web interface.

The bot interacts with users using the Bot API. Management of bots on the platform is available in the bot BotFather. In particular, there one can create a bot and view or change the API token assigned to it

\section{CONCLUSION}

The Internet is that originally was a medium for the transmission of information, now increasingly takes over the functions of the communicator. The global network is becoming a special environment of communication that occupies an important place in all spheres of society.

To date, the problem of the virtual online communication has become popular as a result opportunity for fast access to information, simultaneous work in the network of many users, collection and exchange of various information, to support training and retraining, communication to address personal and business issues with clients and partners, conducting various analytical studies and other benefits.

That is why during these technologies chatbots and recommendation systems are so popular in all areas of our lives.

Dialogue recommendation systems bring obvious benefits to owners of online stores, various services and applications. They recommend the user exactly what he is interested in and generate profits.

In other cases, they can bring not only material benefits. Recommended dialogue systems can help choose a profession, machine, or perfume as it was mentioned above.

That is why such a recommendation dialog system for selecting the computer hardware configuration has a right to exist, and will be very useful in its field.

\section{REFERENCES}

[1] Hill, J., Ford, W. and Farreras, I. (2015). Real conversations with artificial intelligence: a comparison between humanhuman online conversations and human-chatbot conversations. Comput. Hum. Behav., 49, pp.245-250.

[2] Melville, P., Mooney, R., and Nagarajan, R. (2002). Content-Boosted Collaborative Filtering for Improved Recommendations. Eighteenth National Conference on Artificial Intelligence, pp.187-192.

[3] Ricci, F., Rokach, L. and Shapira, B. (2015). Recommender Systems: Introduction and Challenges. In: Ricci F., Rokach
L., Shapira B. (eds) Recommender Systems Handbook. Springer, Boston, MA.

[4] Sarwar, B., Karypis, G., Konstan, J. and Riedl, J. (2001). Item-based Collaborative Filtering Recommendation Algorithms, 10th International Conference on the World Wide Web, New York, pp. 285-295.

[5] Lu, Z., Dou, Z., Lian, J., Xie, X. and Yang, Q. (2015). Content-Based Collaborative Filtering for News Topic Recommendation. AAAI Conference on Artificial Intelligence, 29(1), pp 162-167.

[6] Folstad, A. and Brandtzaeg, P.B. (2017). Chatbots and the new world of HCI. Interactions, 24(4), pp.38-42.

[7] Janarsanam, S. (2017). Hands-On Chatbots and Conversational UI Development. Packt Publishing Ltd, Mumbai Maharastra.

[8] Dewes, C., Wichmann, A. and Feldmann, A. (2003). Ananalysis of Internet chat systems, Proc. ACM SIGCOMM IMC, pp. 51-64.

[9] Bots: An introduction for developers. Available at: https://core.telegram.org/bots [Accessed 12 June 2021].

[10] Sutikno, T., Handayani, L., Stiawan, D., M. Riyadi, M. and Subroto, I. (2016). WhatsApp Viber and Telegram which is best for instant messaging?, International Journal of Electrical and Computer Engineering, 6(3), pp. 909-914.

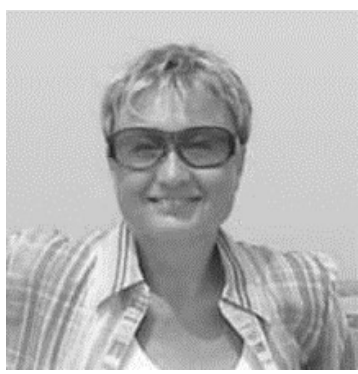

Iryna Yurchak. She is a candidate of Technical Sciences, Associate Professor of the Department of Automated Design Systems, Institute of Computer Science and Information Technologies, Lviv Polytechnic National University. 1963.

Date of birth: August 7,

Education:

Lviv Polytechnic Institute, Faculty of Automation, 1987;

Postgraduate studies, Lviv Polytechnic Institute, 1987-1992;

Candidate's dissertation "Synthesis and application of neural networks with projective and ordered lateral connections", State Research Institute of Information Infrastructure, Lviv, 1999.

Professional activity:

1987-1992 - a graduate student of Lviv Polytechnic Institute

1995 - 2001 - an engineer of the ACS department;

2000 - 2002 - a Senior Lecturer of the Department of Computer Science, Lviv Polytechnic National University;

2001 - 2002 - a Senior Lecturer of the Department of CAD; 2002 - present - an Associate Professor of CAD.

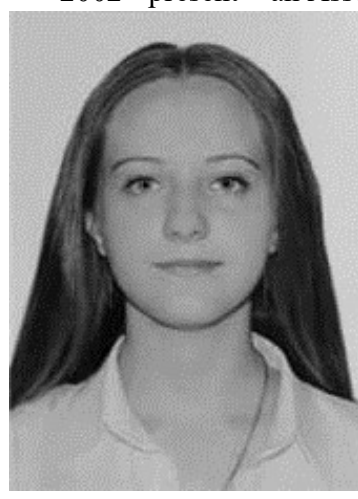

Oksana Svystun. A fourthyear computer engineering student at Lviv Polytechnic National University.

In 2021 she received a bachelor's degree in the specialty. She is interested in studying web technologies and plans to work as a Front-End developer. To achieve her goals, she completed the Front-End Basic course and is currently enrolled in the FrontEnd Advanced course at CURSOR Education. Her hobbies

are cycling and drawing. 